\title{
Methyltransferase Activity of Dengue RNA Virus by Using Purified Protein
}

 \\ Ishtiaq Qadri ${ }^{1}$ \\ ${ }^{1}$ Department of Biological Sciences, Faculty of Science, King Abdulaziz University, Jeddah 21589, Saudi Arabia \\ ${ }^{2}$ Department of Applied Biochemistry, Faculty of Sciences, University of Jeddah 23445, Jeddah Saudi Arabia \\ *Corresponding author: Afaf S Alwabli, Department of Biological Sciences, Faculty of Science, King Abdulaziz University, Jeddah, Saudi Arabia, Tel: +966531937700 \\ E-mail: afafalwabli@yahoo.com
}

Received March 26, 2019; Accepted April 30, 2019; Published June 11, 2019

\begin{abstract}
Dengue disease (DENV) is the purpose behind dengue fever. It is a mosquito-borne single positive-stranded RNA contamination of the family Flaviviridae; sort Flavivirus. Dengue illness is a mosquito-borne making or re-rising pathogen. Its positive sense RNA genome has a top at the 5'- end and no poly(A) tail at the 3'- end. The viral RNA encodes a single polyprotein, C-M-E-NS1-NS2A-NS2B-NS3-NS4A-NS4B-NS5. The polyprotein is prepared into three auxiliary proteins (C, M, and E) and seven nonstructural (NS) proteins (NS1, NS2A, NS2B, NS3, NS4A, NS4B, NS5). NS3 and NS5 are multifunctional chemicals performing different undertakings in the viral life cycle. The N-terminal area of NS5 has unmistakable GTP and S-adenosylmethionine (SAM) restricting locales. The job of GTP restricting site is trapped in the guanylyltransferase (GTase) movement of NS5. We depict the development of the MTase space of NS5 in an E. coli articulation vector and conditions for enzymatic activity of N7-and 2'O-methyltransferase exercises that yield the last sort I 5'- topped RNA.
\end{abstract}

Keywords: DENV; Flavivirus; Methyltransferase; S-adenosylmethionine (SAM); Nonstructural (NS) proteins

\section{Introduction}

Dengue is transmitted by Aedes mosquitoes, essentially Aedes aegypti [1], and alternatively by Aedes albopictus. In tropical and subtropical zones, Aedes aegypti is a significant contributing component in spreading dengue disease for reasons as seeks after; 1) this mosquito likes to breed in clean torpid water close nuclear family zones; 2) it perhaps takes a blood supper from various human hosts; and 3) the mosquito is fit for completing its life cycle in shorter period (reviewed by Wilder-Smith et al. and Juliano [2,3]). An unnatural climate change is foreseen to energize the spread of the mosquito vector. Aedes albopictus, on the other hand, is responsible for the contamination spread in cool airs. In the previous information was open to make convincing treatment. In any case, by and by with types of progress in sub-nuclear science, the sub-nuclear instruments essential the DENV replication cycle have been unraveled. In this way, various potential protein targets and their abilities are directly known. One such potential target is DENV methyltransferase, which has been through and through affirmed [46]. Using nuclear encounters of protein structure and limit, capable computational methodologies are responsible for the progression made in the drug disclosure in current time. Organized essential information is open for all of the dengue viral proteins. Among all, DENV MTase has earned the most outrageous thought of experts starting late.

The flavivirus RNA is enlivened with a saved sort 1 top (N7meGpppA2'Ome-RNA) at its $5^{\prime}$-end, an extraordinary structure comprising of a reversed guanosine connected to the central translated RNA nucleotide by a $5^{\prime}-5^{\prime}$ triphosphate extension. Viral MTases are associated with the mRNA topping procedure, exchanging a methyl amass from the cofactor S-adenosyl-1-methionine (AdoMet) onto the $\mathrm{N} 7$ particle of the top guanine and onto the $2^{\prime} \mathrm{OH}$ gathering of the ribose moiety of the first RNA nucleotide. In the variety Flavivirus, both (guanine-N7)-methyltransferase (N7MTase) and (nucleoside-2' O-)-methyltransferase (2'OMTase) exercises have been related with the $\mathrm{N}$-terminal area of the viral NS5 protein $[5,7,8]$. The NS proteins join the essential, significantly spared proteins NS1, NS3, and NS5, and the four minimal hydrophobic, less directed, NS2A, NS2B, NS4A, and NS4B proteins30. The getting ready of the DENV polyprotein is finished coand post-translationally by a mix of cell proteases of the furin-type or other Golgi-bound proteases and the viral serine protease NS3, which requires NS2B for its development [9]. The RNA triphosphatase and guanylyl-transferase exercises are situated in the $\mathrm{N}$-terminal space of D1 $[10,11]$, though the guanine N7-MT active site is in the C-terminal area of D1 [12]. In any case, the MT space requires a relationship with the D12 subunit for full movement, having an original action 40-multiple times not as much as that of the D1-D12 heterodimer [13,14]. The D12 subunit demonstrates no arrangement likeness with some other realized protein but to homologs present in different poxviruses. The D1-D12 heterodimer likewise assumes a job in translation commencement of moderate qualities [15] just as interpretation end of new qualities of vaccinia infection [16]. Vaccinia viral mRNAs secure the top 1 structure by $2^{\prime}$-O-methylation of the principal interpreted nucleotide by the VP39 protein, which is additionally a processivity subunit for the viral poly-A polymerase (VP55) [17].

\section{Materials and Methods}

\section{Materials}

DNA polymerase, T4 DNA ligase and restriction enzymes used in all routine cloning and transformation experiments were procured from New England Biolabs Inc. MA, USA (NEB). Analytical and molecular biology products including agarose, antibiotics, and enzymes like proteinase $\mathrm{K}$, lysozyme and other chemical reagents were purchased from Sigma, USA. Plasmid isolation kit, cDNA synthesis kit, and quick gel extraction kit were procured from Qiagen, Thermo scientific and Invitrogen.

\section{Bioinformatics analysis}

The FASTA arrangements of the recuperated progressions were used for the area association. Methyltransferase area contains 262 amino acids, and the unique field was physically apportioned and asserted by utilizing Pfam, Prosite and joined programming, InterProScan. 


\section{Cloning of NS5 methyltransferase domain}

To clone the NS5 methyltransferase gene sequence was retrieved from PubMed and primers were designed to amplify the NS5 methyltransferase region expected PCR size is 786 base pair amplifying NS5 methyltransferase domain for dengue virus independently. NS5 methyltransferase gene contains introns; therefore it was amplified from the cDNA, and cloned cDNA was set up from the culture utilizing fitting unit, and it encodes amino acid 262 amino acid. The purified PCR products were subjected to ligation into the pGEMT easy vector for T-A cloning. The ligated mixture was transformed into DH5a E. coli cells and was placed on LB-Agar containing IPTG and X-gal. The white colonies were screened for positive clone by restriction digestion and by colony PCR as well. After restriction digestion, NS5 methyltransferase gene inserts of positive clones were purified from agarose gel and were subjected to standard ligation into an expression vector (pET28a+) (Novagen, Madison, WI, USA) and then transformed into E. coli DH5a. The plasmids of recombinant colonies were purified and confirmed by restriction digestion analysis.

\section{Expression and purification of recombinant protein}

The pET28a+ expression clones were transformed into E. coli strains BL21 codon plus. 1.0 mM IPTG induced the overexpression of recombinant proteins. The harvested culture pellet was resuspended into bacterial lysis buffer of pH $7.8(20 \mathrm{~mm}$ Tris- $\mathrm{HCl}, 250 \mathrm{~mm} \mathrm{NaCl}, 0.1 \%$ Tween 20, $0.1 \%$ Triton 100 and the protease inhibitor cocktail from Sigma (St. Louis, MO, USA) and sonicated to lyse the maximum number of cells. The soluble fraction was separated after centrifugation and allowed to bind with equilibrated Ni-NTA (Qiagen, GmbH, Germany) in the binding buffer (20 mm Tris- $\mathrm{HCl} \mathrm{pH} \mathrm{8.0,} 250 \mathrm{~mm} \mathrm{NaCl}, 5 \mathrm{mM}$ imidazole and protease inhibitor cocktail (Sigma, St. Louis, MO, USA) for one and half hour at $4^{\circ} \mathrm{C}$. The column was stringently washed with excess wash buffer (10-20 mm Tris- $\mathrm{HCl} \mathrm{pH} \mathrm{8.0,} 250 \mathrm{~mm} \mathrm{NaCl}, 25-50 \mathrm{mM}$ imidazole and protease inhibitor cocktail) to remove the non-specifically bounded protein from Ni-NTA. The recombinant His-tag protein was eluted with varying concentration of imidazole $(50-250 \mathrm{~mm})$ in elution buffer $(20$ $\mathrm{mm}$ Tris- $\mathrm{HCl} \mathrm{pH}$ 8.0, $250 \mathrm{~mm} \mathrm{NaCl}, 10 \%(\mathrm{v} / \mathrm{v})$ glycerol and protease inhibitor cocktail (Sigma, St. Louis, MO, USA). Purified protein fraction was further subjected to SDS-PAGE and western blot to check the purity of proteins. The purified recombinant proteins were used for the rest of the experiments.

\section{Western blot analysis}

The purified proteins were transferred from the SDS-PAGE gel onto nitrocellulose membrane or PVDF. Transfer of the purified proteins to the membrane was checked by using PonceauS staining before the blocking. The blot was blocked in blocking buffer (3\% BSA) for 2, and subsequently, it was washed and incubated for 1 hour with primary antihis tag antibody (1:3000). The blot was then removed and incubated for 1 hour with appropriate secondary antibody (1:5000) conjugated to alkaline phosphatize or horseradish peroxidize (HRP) and incubate at room temperature for 2 hours. The blots were developed by using of ECL kit. The reaction was stopped by rinsing the stain in $10 \mathrm{~mm}$ EDTA, pH 8.0.

\section{Enzymatic assay}

This convention portrays an in vitro measure for histone methyltransferase action that utilizes bacterial cell removes in which articulation of a methyltransferase of intrigue is incited. Methyltranferase substrate of interest other than S-adenosyl-1-methionine (SAM). 96well, 384-well, low-volume 384-well or 1,536-well white assay plate with a solid bottom (Do not use black plates or clear plates). The final concentration of SAM in the methyltransferase reaction will be $10 \mu \mathrm{M}$ (because this is a $2 \mathrm{X}$ reaction $\mathrm{mix}$ ). You may also perform assays at a final SAM concentration of $1 \mu \mathrm{M}$ by preparing the $2 \mathrm{X}$ substrate with 2 $\mu \mathrm{M}$ SAM (i.e., $2 \mu \mathrm{l}$ of $1 \mathrm{mM}$ SAM per $1 \mathrm{ml}$ of the $2 \mathrm{X}$ substrate). To use SAM at a different final concentration, adjust the volume of $1 \mathrm{mM} \mathrm{SAM}$ accordingly. 96-well plate for enzyme dilution and keep it on a shaker. Plate-reading illuminometer. Assay protocol was performed as described in Promega kits. Determine the optimal enzyme concentration for your subsequent experiments by plotting luminescence (Y-axis) against enzyme concentration (X-axis) using GraphPad Prism ${ }^{\circledR}$ or similar software [18-20].

\section{Results and Discussion}

\section{Investigation of amino acid arrangement}

We have dissected the corrosive amino succession of the methyltransferase space (262 amino acids) by utilizing unique, accessible instruments of bioinformatics. The outcome appeared in Figure $1 \mathrm{~A}$ is the different critical space of Dengue infection (DEV) including NS5 methyltransferase. The InterProScan examination shows that methyltransferase (MTs) establish domain details (Figure 1A). Structure assessment and residue numbers were identified by using the Swiss model server (Figure 1B and 1C).

\section{Cloning, expression and purification of methyltransferase 5}

The PCR amplified fragments were successfully cloned in pGEMT and then sub-cloned into a pET28a+ expression vector (Figure $2 \mathrm{~A}-2 \mathrm{C}$ ).

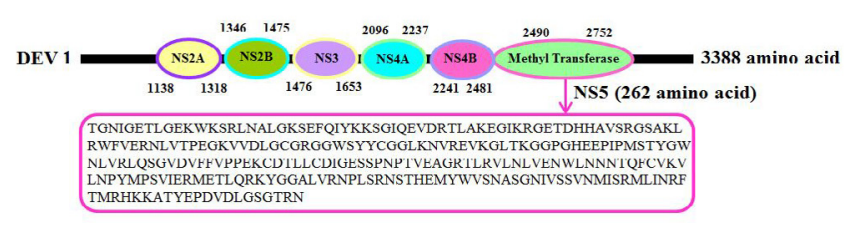

Figure 1A: The specific domain organization of Dengue virus. The conserved sequences of each important domain are written inside the boxes and highlighted the methyltransferase domain. The text in blue color refers to the names of several conserved domains and the numbers refer to the amino acids sequence separating the various areas.

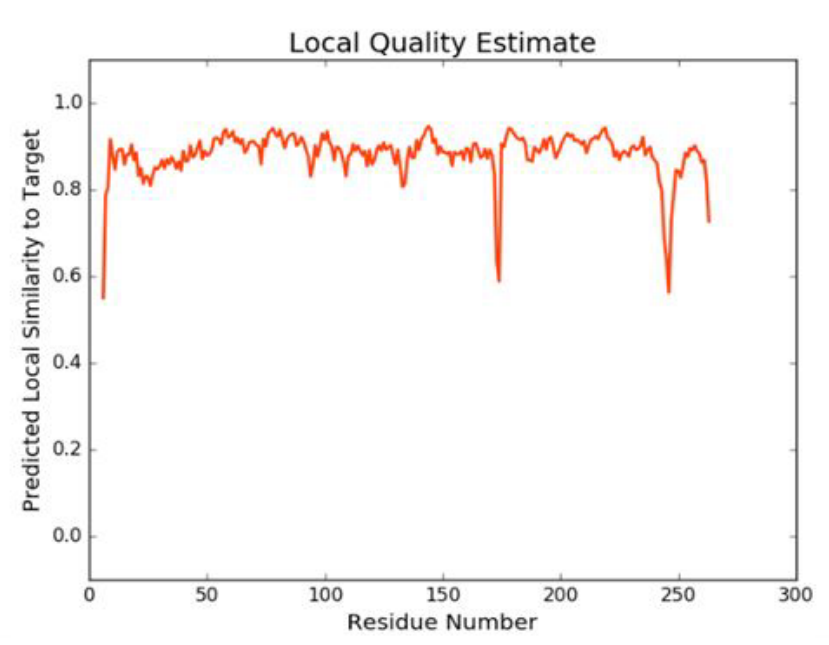

Figure 1B: Structure assessment and residue numbers. 


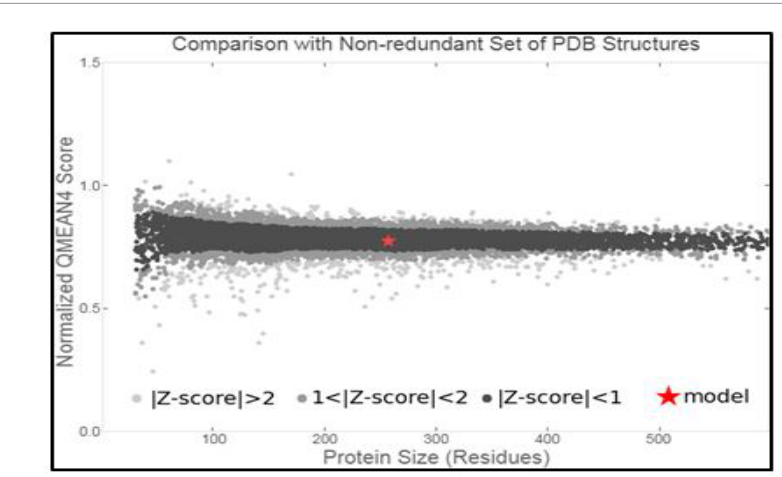

Figure 1C: Comparison with a non-redundant set of PDB structure, protein size residue.



Figure 2A: Amplification of methyltransferase gene. Amplified PCR product of methyltransferase gene Lane 1 contain amplified product and lane $\mathrm{M}$ contain 1 $\mathrm{Kb}$ DNA ladder.

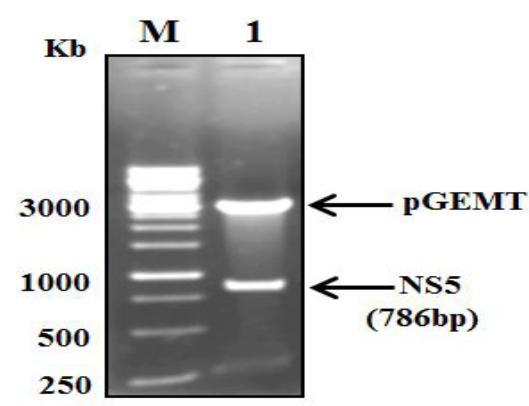

Figure 2B: The PCR products of all the fragments were cloned in pGEMT Easy vector. Lanes-1 shows the restriction enzyme digestion of plasmid of positive clones corresponding to of methyltranferase. Lane M contains $1 \mathrm{~Kb}$ DNA ladder.

The overexpression of methyltransferase protein in E. coli strain BL21 codon plus was induced with $1 \mathrm{mM}$ IPTG at $37^{\circ} \mathrm{C}$ and the significant fraction of expressed protein was found in the soluble fraction. Thus all the three proteins were purified under native conditions using $\mathrm{Ni}$ NTA affinity chromatography. The SDS-PAGE analysis of the purified proteins showed that $29 \mathrm{kDa}$ protein (Figure 2D). The pure fractions of methyltransferase were pooled and then subjected to another round of purification using Ni-NTA affinity chromatography. One of the portions of eluting showed profoundly real band (Figure 2D), and this fraction was used for the various enzymatic activities. The purified fractions were further checked by western blot analysis using anti-his antibodies and only a single band in each of the purified fraction was detected (Figure 2E).

\section{Methyltranferase assay}

For instance, the Set1 histone methyltransferase, from S. cerevisiae,

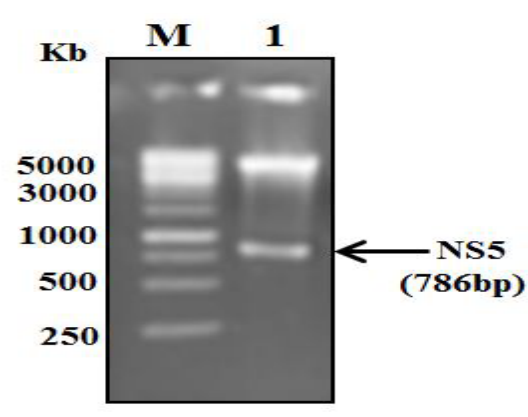

Figure 2C: The fragment excised from pGEMT and Sub-cloning of genes into pET28a expression vector at the appropriate sites. Lane-1 show positive clones of methyltranferase in expression vector pET28a. Lane M contains $1 \mathrm{~Kb}$ DNA ladder.



Figure 2D: Purification of methyltransferase. SDS page gels of purified proteins. Lane 1 and 2 contain of the purified proteins ( $29 \mathrm{kDa})$ and lane $\mathrm{M}$ contains the protein molecular weight marker (prestained).

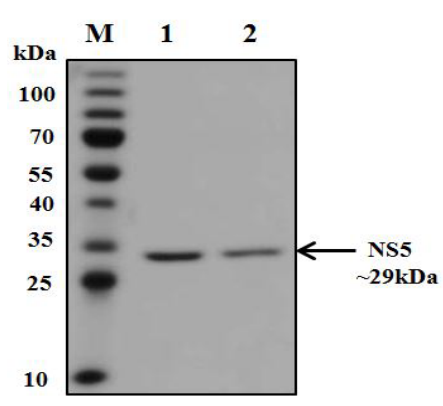

Figure 2E: Western blot of purified protein; lane1 and 2 contain purified protein. Lane $\mathrm{M}$ in all the above blots denotes protein molecular weight marker (prestained).

is just dynamic when it is available in a multiprotein complex [2124]. Reconstitution of these protein buildings in vitro or cleansing of the complex from cells might be necessary for watching histone methyltransferase movement. Generally speaking, this strategy fills in as a beginning stage for the underlying distinguishing proof and portrayal of conceivably novel histone methyltransferase and, with different changes, can be utilized to analyze other histone and nonhistone specific enzymatic exercises. Histone methyltransferase catalyze the expansion of at least one methyl gatherings to a particular lysine or arginine buildup inside histones. The rate of the reaction is determined using the 3, 5-dichloro-2-hydroxybenzene sulfonic acid (DHBS) extinction coefficient $15.0 \mathrm{mM}$. One unit of methyltransferase will transfer $1.0 \mu \mathrm{mol}$ of a methyl group per minute at $37^{\circ} \mathrm{C}$. Calculate the change in absorbance points on the linear portion of the curve (Figure 
3A). We also deign plan of the methylation assay. The removal of the methyl group from SAM generates S-adenosylhomocysteine (AdoHcy), which is rapidly converted to S- ribosyl homocysteine and adenine by the included AdoHcy nucleosidase (Figures 3B and 4).

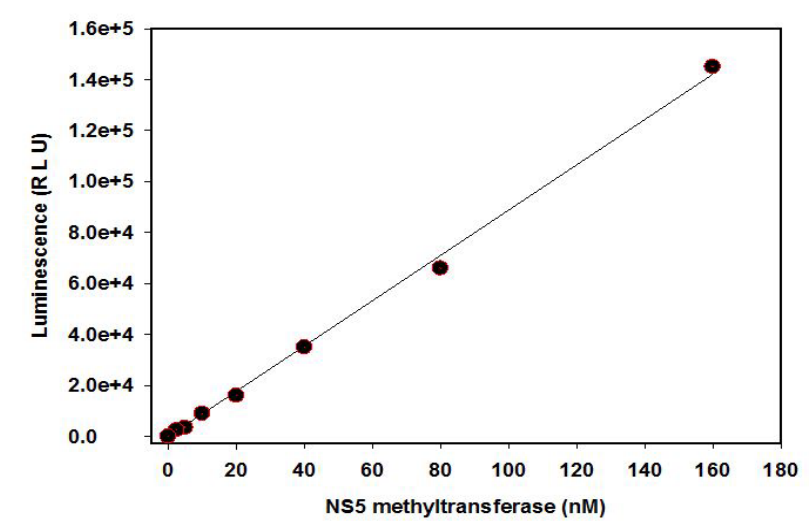

Figure 3A: Methyltranferase assay in the presence of NS5 purified proteins.

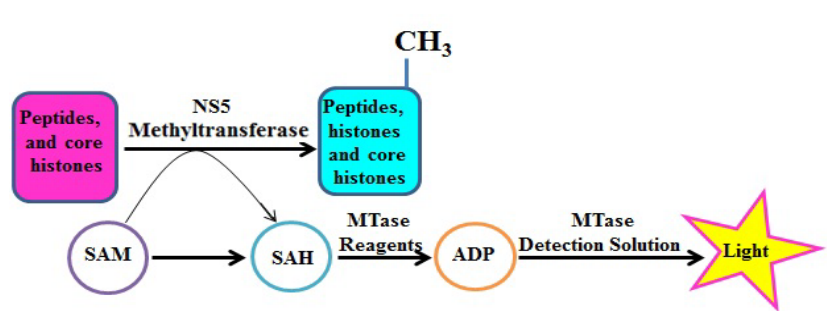

Figure 3B: Frameworks the general scheme of the assay. The removal of the methyl group from SAM generates S-adenosylhomocysteine (AdoHcy), which is rapidly converted to $S$ - ribosyl homocysteine and adenine by the included AdoHcy nucleosidase.

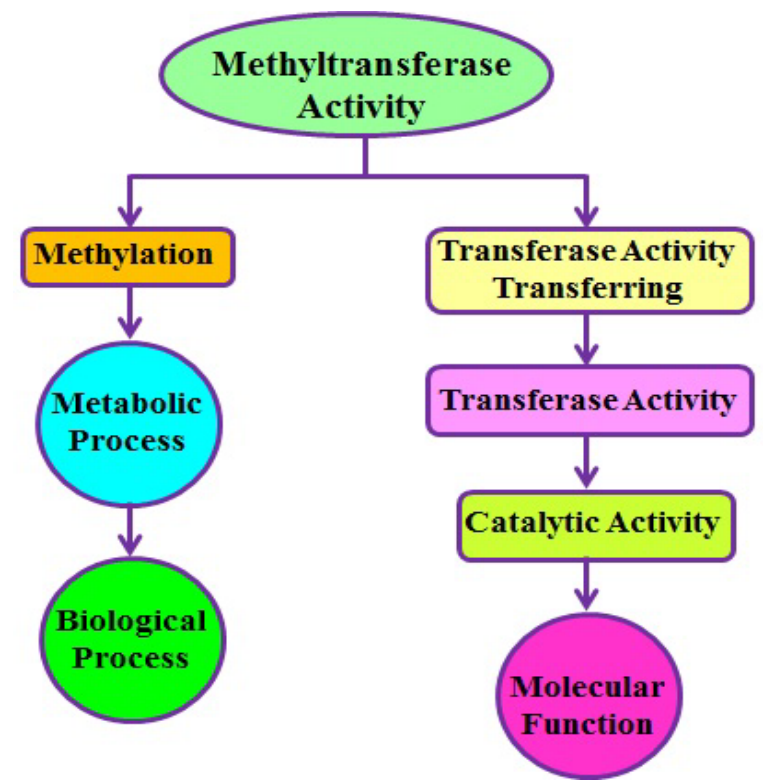

Figure 4: Projected the model for mechanism of methyltranferase action.

\section{Conclusion}

Dengue infection (DENV) protein NS5 conveys two mRNA top methyltransferase (MTase) exercises associated with the combination of a top structure, $(7 \mathrm{Me}) \mathrm{GpppA}\left(2^{\prime} \mathrm{OMe}\right)-\mathrm{RNA}$, at the 5 - end of the viral mRNA. The methylation of the top guanine at its N7-position (N7MTase, (7Me)GpppA-RNA) is fundamental for viral replication. The characterization of NS5 methyltranferase with intrinsic Methylation activities and possible role in replication related may offer an important contribution to our better understanding of metabolic processes in the dengue virus.

\section{Acknowledgments}

Authors are grateful to King Abdulaziz City for Science and Technology (KACST, Riyadh, Saudi Arabia), General Directorate of the research grants program for funding this study by grant No.(1-18-01-009-0035). This work was also funded by the Deanship of Scientific Research (DSR) grant No.(), King Abdulaziz University, Jeddah, Saudi Arabia, The authors, therefore, acknowledge with thanks DSR technical and financial support.

\section{References}

1. Halstead SB (2008) Dengue virus-mosquito interactions. Ann Rev Entomol 53: 273-291.

2. Wilder-Smith A, Ooi EE, Vasudevan SG, Gubler DJ (2010) Update on dengue: Epidemiology, virus evolution, antiviral drugs, and vaccine development. Current Infect Dis Rep 12: 157-164.

3. Juliano R (2002) Signal transduction by cell adhesion receptors and the cytoskeleton functions of integrins, cadherins, selectins, and immunoglobulin-superfamily members. Ann Rev Pharma Toxic 42: 283-323.

4. Dong H, Chang DC, Xie X, Toh YX, Chung KY, et al. (2010) Biochemical and genetic characterization of dengue virus methyltransferase. Virology 405: 568-578.

5. Zhou Y, Ray D, Zhao Y, Dong H, Ren S, et al. (2007) Structure and function of flavivirus NS5 methyltransferase. J Vir 81: 3891-3903.

6. Dong H, Zhang B, Shi PY (2008) Flavivirus methyltransferase: A novel antiviral target. Antivir Res 80: 1-10.

7. Egloff MP, Benarroch D, Selisko B, Romette JL, Canard B (2002) An RNA cap (nucleoside-2'-O-)-methyltransferase in the flavivirus RNA polymerase NS5: Crystal structure and functional characterization. The EMBO J 21: 2757-2768.

8. Ray D, Shah A, Tilgner M, Guo Y, Zhao Y (2006) West Nile virus $5^{\prime}$-cap structure is formed by sequential guanine $\mathrm{N}-7$ and ribose $2^{\prime}-\mathrm{O}$ methylations by nonstructural protein 5. J Vir 80: 8362-8370

9. Bollati M, Alvarez K, Assenberg R, Baronti C, Canard B, et al. (2010) Structure and functionality in flavivirus NS-proteins: Perspectives for drug design. Antivir Res 87: 125-148.

10. Niles EG, Christen L (1993) Identification of the vaccinia virus mRNA guanyltransferase active site lysine. J Biol Chem 268: 24986-24989.

11. Myette JR, Niles EG (1996) Characterization of the Vaccinia virus RNA $5^{\prime}$-triphosphatase and nucleoside triphosphate phosphohydrolase activities demonstration that both activities are carried out at the same active site. J Biol Chem 271: 11945-11952.

12. Cong P, Shuman S (1992) Methyltransferase and subunit association domains of vaccinia virus mRNA capping enzyme. J Biol Chem 267: 16424-16429.

13. Higman M, Bourgeois N, Niles E (1992) The vaccinia virus mRNA (guanine-N7-)methyltransferase requires both subunits of the mRNA capping enzyme for activity. Journal of Biological Chemistry 267: 16430-16437.

14. Mao X, Shuman S (1994) Intrinsic RNA (guanine-7) methyltransferase activity of the vaccinia virus capping enzyme D1 subunit is stimulated by the D12 subunit. Identification of amino acid residues in the D1 protein required for subunit association and methyl group transfer. Journal of Biological Chemistry 269: 24472-24479.

15. Vos JC, Sasker M, Stunnenberg HG (1991) Vaccinia virus capping enzyme is a transcription initiation factor. The EMBO J 10: 2553-2558.

16. Shuman S, Moss B (1988) Factor-dependent transcription termination by vaccinia virus RNA polymerase. Evidence that the cis-acting termination signal is in nascent RNA. J Biol Chem 263: 6220-6225. 
Citation: Alwabli AS, Alattas SG, Alhebshi AM, Zabermawi NM, Alkenani N, et al. (2019) Methyltransferase Activity of Dengue RNA Virus by Using Purified Protein. Int J Drug Dev \& Res 11: 16-20

17. Schnierle BS, Gershon PD, Moss B (1992) Cap-specific mRNA (nucleoside-O2'-)methyltransferase and poly (A) polymerase stimulatory activities of vaccinia virus are mediated by a single protein. Proceed Nation Acad Sci 89: 2897-2901.

18. Fauman EB, Blumenthal RM, Cheng X (1999) Structure and evolution of AdoMet-dependent methyltransferases. In: S-Adenosylmethionine-dependent methyltransferases: structures and functions. World Sci, pp: 1-38.

19. Schubert HL, Blumenthal RM, Cheng X (2003) Many paths to methyltransfer: A chronicle of convergence. Trends Biochem Sci 28: 329-335.

20. Dorgan KM, Wooderchak WL, Wynn DP, Karschner EL, Alfaro JF, et al. (2006) An enzyme-coupled continuous spectrophotometric assay for S-adenosylmethioninedependent methyltransferases. Analyt Biochem 350: 249-255.
21. De Vreede GJ, Briggs RO (2001) Thinklets: Five examples of creating patterns of group interaction. Group Decision \& Negotiation, La Rochelle, France, pp: 199-208.

22. Krogan NJ, Dover J, Khorrami S, Greenblatt JF, Schneider J, et al. (2002) COMPASS a histone $\mathrm{H} 3$ (Lysine 4) methyltransferase required for telomeric silencing of gene expression. J Biol Chem 277: 10753-10755.

23. Kalmár-Nagy T, Ganguly P, D'Andrea R (2002) Real-time trajectory generation for omnidirectional vehicles. In: Proceedings of the 2002 American control conference (IEEE Cat No CH37301) 2002: 286-291.

24. Roguev A, Schaft D, Shevchenko A, Pijnappel WP, Wilm M, et al. (2001) The Saccharomyces cerevisiae Set1 complex includes an Ash2 homologue and methylates histone 3 lysine 4. The EMBO J 20: 7137-7148. 